\title{
Anomalous energy consumption detection using a Naïve
}

\section{Bayes approach [version 1; peer review: 1 approved with}

\section{reservations]}

\author{
Jia Yan Lim (D), Wooi-Nee Tan, Yi-Fei Tan (iD)
}

Faculty of Engineering, Multimedia University, Cyberjaya, Selangor, 63100, Malaysia

V1 First published: 19 Jan 2022, 11:64

https://doi.org/10.12688/f1000research.70658.1

Latest published: 19 Jan 2022, 11:64

https://doi.org/10.12688/f1000research.70658.1

\section{Abstract}

Background: Industrial energy management has emerged as an important component in monitoring energy consumption particularly with the recent trend of migrating towards IR 4.0. The capability to detect anomalies is essential as it serves as a precautionary step for real-time response to mitigate the maximum demand penalty. The purpose of this research was to develop a high accuracy anomalies detection algorithm to identify anomalies in the energy consumption data recorded by a smart meter.

Methods: The proposed algorithm utilized supervised and unsupervised machine learning techniques, namely Isolation Forest and Gaussian Naïve Bayes. The data were first labeled by using Isolation Forest to categorize them into normal and abnormal groups. This was followed by Gaussian Naïve Bayes to classify and predict the anomalies of the smart meter reading.

Results: These machine learning techniques showed significant accuracy in predicting the anomalies in smart meter readings. The data used were simulated data collected in less than a month with 30minute reading intervals. The data were divided into testing and validation sets according to a ratio of 7:3. The balanced accuracy score in predicting anomalies for each different smart meter was above $89 \%$. The average precision, average recall and average F1 score for the normal data were $98 \%, 99 \%$ and $98 \%$, respectively. Whereas the corresponding scores for the abnormal data set were $95 \%, 90 \%$ and $92 \%$.

Conclusions: The proposed algorithm is a hybrid approach based on Isolation Forest and Gaussian Naïve Bayes and it provided satisfactory accuracy in anomaly electricity consumption detection based on smart meter readings. The study presents a quick and simple method for categorizing energy consumption data as normal or abnormal, which assists in automatically labelling vast datasets of energy consumption readings. The proposed approach establishes a fundamental framework for predicting the occurrence of anomalies in the industrial

\section{Open Peer Review}

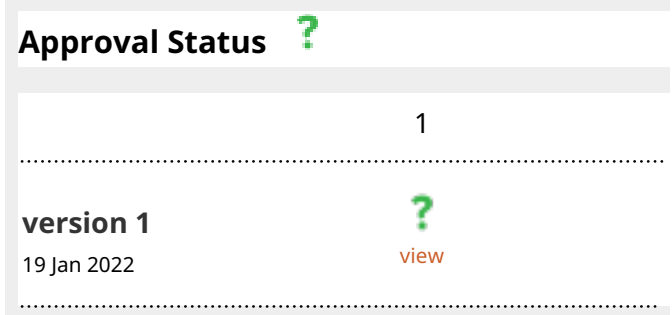

1. Kim Gaik Tay (D), Universiti Tun Hussein Onn Malaysia, Batu Pahat, Malaysia

Any reports and responses or comments on the article can be found at the end of the article. 
energy management system.

Keywords

anomaly detection, energy consumption, data labeling, Isolation

Forest, Gaussian Naïve Bayes, energy management, machine learning

techniques

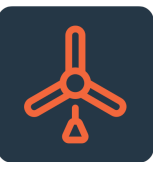

This article is included in the Energy gateway.

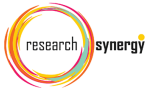

This article is included in the Research Synergy

Foundation gateway.



This article is included in the Artificial

Intelligence and Machine Learning gateway.

Corresponding author: Yi-Fei Tan (yftan@mmu.edu.my)

Author roles: Lim JY: Data Curation, Investigation, Software; Tan WN: Conceptualization, Methodology; Tan YF: Methodology, Software Competing interests: No competing interests were disclosed.

Grant information: The author(s) declared that no grants were involved in supporting this work.

Copyright: @ 2022 Lim JY et al. This is an open access article distributed under the terms of the Creative Commons Attribution License, which permits unrestricted use, distribution, and reproduction in any medium, provided the original work is properly cited.

How to cite this article: Lim JY, Tan WN and Tan YF. Anomalous energy consumption detection using a Naïve Bayes approach [version 1; peer review: 1 approved with reservations] F1000Research 2022, 11:64 https://doi.org/10.12688/f1000research.70658.1

First published: 19 Jan 2022, 11:64 https://doi.org/10.12688/f1000research.70658.1 


\section{Introduction}

People nowadays are continuously looking for new ways to utilize energy to improve their lives, therefore the demand for it is increasing. Most of the time, companies and industries struggle to monitor all their devices at the same time, which can lead to power wastage at any time. As a result, operational expenses will be greater than necessary. Besides that, power wastage contributes to global warming by releasing carbon when energy is generated through burning coal, gas, and oil. Hence, solutions are required to address these issues. Energy Management Information Systems (EMIS) ${ }^{1}$ or Building Energy Management Systems (BEMS) ${ }^{2}$ are the tools for continuous energy management monitoring that collect and analyze energy data on a regular basis. However, since no fixed characteristics can be retrieved, and no current static model of energy can be referred to, they give a low accuracy in predicting electricity consumption. According to an article published in Energy Malaysia, ${ }^{3}$ consumers may use the information supplied by smart meters to assist them to take prompt action in terms of power usage and waste. However, a model which is able to give early detection of deviations from historical energy usages is important and beneficial to multiple parties.

Many authors have conducted research on detecting the abnormal behavior of power usage. Yassine Himeur et al., ${ }^{4}$ used a rule-based model to extract micro-moment features. In their method, the accuracy varied from $93.91 \%$ to $99.58 \%$ depending on the types of datasets. The main limitation of their proposed work was related to the use of supervised learning in training the classifier. Wenqiang Cui and Hao Wang ${ }^{5}$ proposed a hybrid model that combines polynomial regression and Gaussian distribution to detect the anomalies of data. They focused on school power consumption and were able to detect anomalies with 0 false-negative and an average precision higher than $91 \%$. However, the model must be trained manually. Ma and $\mathrm{Zhang}^{6}$ developed a method to detect abnormal building energy consumption in real time. They used fractal correlation dimension (FCD) and proper orthogonal decomposition linear stochastic estimation (PODLSE). They claimed that the performance of the method depended on the threshold selected. Thus, improvement was required by integrating with other intelligent algorithms. Jecinta Mulongo et al., ${ }^{7}$ used support vector machines (SVM), K-Nearest Neighbors (KNN), Logistic Regression (LR), and MultiLayer Perceptron (MLP) to detect anomalies in power generation plant data. They reported that MLP has the best performance in the evaluation measurement with a score of $96 \%$ in the K-fold cross validation test. In addition, deep learning has also received increasing interest for abnormality detection. ${ }^{8-10}$ Besides using machine learning and deep learning to detect anomaly electricity consumption, statistical techniques are used to monitor the consumption pattern. For example, Lin and Claridge ${ }^{11}$ used the deviation between collected and simulated energy usage and also the standard deviation of the residuals to detect the abnormal consumption. The limitation here was that only limited assumed cases were used. Araya et al., ${ }^{12}$ used sliding window framework to capture contextual characteristics and historical sensor data in detecting the anomalous pattern. Liu et al., ${ }^{13}$ proposed a statistical-based online detection technique with a Lambda scheme, which involved an in-memory distributed computing algorithm. Chen et al. $^{14}$ also proposed a statistical predictive method utilizing mean and variance in detecting the energy anomalies.

One of the challenges that complicates the development of anomaly detection technique is the absence of labelled ground-truth datasets. There is a lack of research addressing how to classify energy consumption readings as normal or abnormal, as well as the nature of the abnormality. ${ }^{15}$ Additionally, as data on electricity consumption continues to expand tremendously, the properties of big data become more apparent, which further complicates the process of detecting anomalies. To that end, this paper proposes a method for detecting anomalies in energy consumption readings using an unsupervised approach that combines Isolation Forest and Naive Bayes models. The Isolation Forest is shown to be capable of detecting not only point anomalies with peaks, but also contextual anomalies. Whereas, when combined with Naive Bayes, the possibility of performing anomaly detection without retraining the data is demonstrated. This hybrid approach to labeling energy consumption readings demonstrates a straightforward and efficient method for assisting in the energy consumption readings labeling, which is critical in today's rapidly growing data volume era.

In this paper, a two-stage anomalous detection model is proposed to detect any anomalies of smart meter readings. This paper is organized as follows: Section 1 is the introduction, section 2 describes the methodology, which includes data labelling and the detection model. Section 3 presents the results and discussion. Finally, we conclude the research and introduce future work in Section 4.

\section{Methods}

The anomalous detection model is built in two stages. The first stage focuses on utilizing the Isolation Forest in labeling the energy consumption data into two categories: normal consumption or abnormal peak. In the second stage, the energy consumption data as well as the corresponding labelled categories are then used to train a detection model using the Gaussian Naïve Bayes approach. The trained model can then be used to detect any anomalous unseen energy consumption data. The proposed model is implemented using the Python programming language, version 3.7 (RRID: SCR_008394). 
Stage 1: Data labeling with Isolation Forest

Isolation Forest is one of the unsupervised machine learning algorithms used to detect anomalies in a dataset. Unlike supervised machine learning algorithms, Isolation Forest does not require any label or classification for the data to be analyzed. The algorithm isolates or separates anomalies by considering anomalies as instances that are less likely to occur or attribute values that are very different from normally attributed values. For Isolation Forest, the anomaly score is calculated with the equation below:

$$
s(x, n)=2^{-\frac{E(h(x))}{c(n)}}
$$

where $h(x)$ is the path length, which is the number of edges where point $x$ meets the decision tree. $E(h(x))$ is the expected number of $h(x)$, and $c(n)$ is the average path length of the total decision tree. From the equation, when the path length is low, an isolated point, the value for $E(h(x))$ is close to zero and the score is close to 1 . When the total path length is equal to the average path length the score will be 0.5 . According to Liu et al., ${ }^{16}$ the potential anomalies can be acknowledged as an anomaly score above 0.6 .

Figure 1 gives the labelling flow chart using Isolation Forest. The unlabeled data was first imported into the Jupyter Notebook (Jupyter Notebook, RRID:SCR_018315). The Isolation Forest from sklearn.ensemble was then used to identify the anomaly scores. In our work, the anomaly scores above 0.53 were classified as abnormal data. The classified values of $C_{0}=0$ were used to indicate normal energy consumption while $C_{1}=1$ indicated anomaly energy consumption.

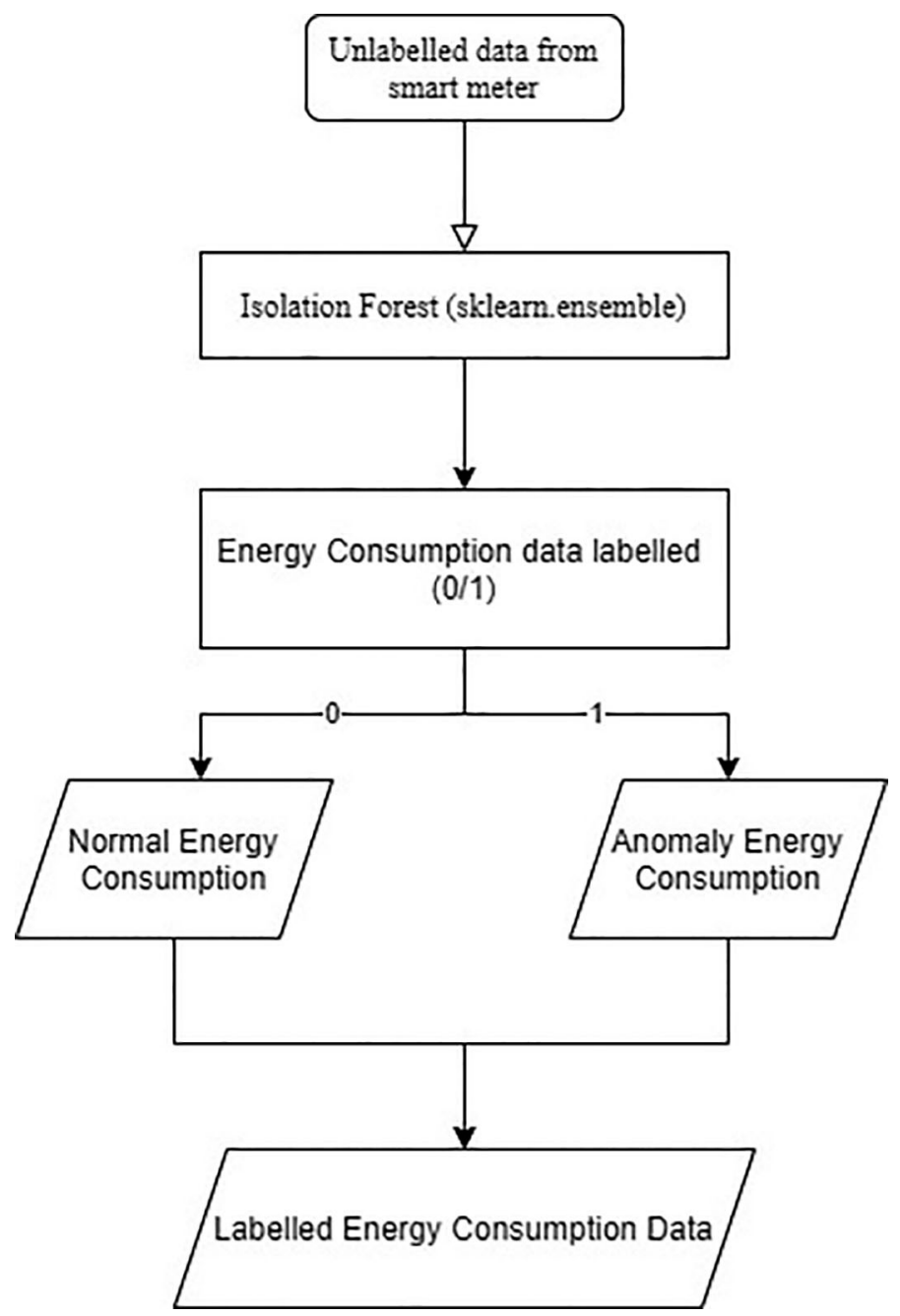

Figure 1. Flow chart of stage 1 data labeling with Isolation Forest. 
Stage 2: Detection model based on Gaussian Naïve Bayes

The Gaussian Naïve Bayes is one of the most popular classifier algorithms in data mining and the data science field. It is well known since it is a simple technique for deciphering binary or categorical input values, and it does not necessitate a large amount of data for training. Since the energy consumption data is in a continuous form, the Gaussian Naïve Bayes that follows a Gaussian normal distribution was chosen to build the detection model. In general, Gaussian Naïve Bayes calculates the probability density of $v$ for certain data to be assigned to respective classes according to the equation:

$$
p\left(x=v \mid C_{k}\right)=\frac{1}{\sqrt{2 \pi \sigma_{k}^{2}}} e^{\frac{-\left(v-\mu_{k}\right)^{2}}{2 \sigma_{k}^{2}}}
$$

with $x$ is the energy consumption, $C_{k}$ is the data labelling, $\mu_{k}$ is the mean of the values in $x$ associated with $C_{k}, \sigma_{k}$ is the Bessel corrected variance of the values in $x$ associated with class $C_{k}$. Thus, given an unseen energy consumption value $x$, its probabilities of being classified into an abnormal class will be calculated based on the well-known Bayesian Theorem:

$$
p\left(C_{1} \mid x\right)=\frac{p\left(x \mid C_{1}\right) p\left(C_{1}\right)}{p(x)}=\frac{p\left(x \mid C_{1}\right) p\left(C_{1}\right)}{\sum_{i=0,1} p\left(x \mid C_{i}\right) \cdot p\left(C_{i}\right)}
$$

with the probabilities $p\left(x=v \mid C_{k}\right)$ can be obtained from equation (1). If $p\left(C_{1} \mid x\right)$ is greater than 0.5 , then the point would be classified as abnormal, else it would be classified as a normal energy consumption point. The trained Naïve Bayes model can then be used to classify the unseen energy consumption reading into normal and abnormal. The proposed anomalous detection model was verified using simulated smart meter readings with each reading for a smart meter collected in 30-minute intervals for a period of 576 hours. Firstly, the pattern of the electricity data of the Irish Social Science Data Archive (ISSDA) were investigated. Next, three sets of smart readings were generated based on different values of peakto-peak and valley-to-valley intervals. Then, the energy consumption values were manually created based on assumption of different users' behavior. ${ }^{17}$

\section{Results}

Three smart meter readings were simulated in the analysis, namely smart meter ID A, smart meter ID B and smart meter ID C. The energy curves of each of these meter readings exhibited varied behavior and their respective energy consumption profiles are depicted in Figure 2. Most of the spikes in the energy curve of smart meter ID $A$ are of similar height. However, the peak values of the energy curve in smart meter ID $B$ are more variable. Meanwhile, the energy curve of smart meter ID $C$ is flatter.

To ensure that the verification process was performed without bias, the data was categorized in a 70:30 ratio, with 70\% of data utilized for training, and 30\% of data reserved for verification as unseen data to the training model. The performance of the anomalous detection model was evaluated using the following formulae on precision, recall and F1 score:

$$
\begin{gathered}
\text { precision }=\frac{\mathrm{TP}}{\mathrm{TP}+\mathrm{FP}} \\
\text { recall }=\frac{\mathrm{TP}}{\mathrm{TP}+\mathrm{FN}} \\
\mathrm{F} 1 \text { score }=2 \cdot \frac{\text { precision } \cdot \text { recall }}{\text { precision }+ \text { recall }}
\end{gathered}
$$

TP, FP and FN refer to true positive, false positive and false negative, respectively.

Take the case when positive $(\mathrm{P})$ refers to the anomalous point, for example, then TP denotes that the abnormal points are accurately identified as abnormal, whereas FP denotes that the normal points are incorrectly detected as abnormal by the model. Finally, abnormal points detected as normal by the model are referred to as FN. Therefore, the precision gives the proportion of detected anomalous points that are actually abnormal. The counterpart of precision is recall, which refers to the ability to recognize abnormal points among the actual abnormal points. F1 score combines precision and recall and calculates the harmonic mean. The F1 score reaches its optimum value of 1 if both precision and recall are both at $100 \%$. 


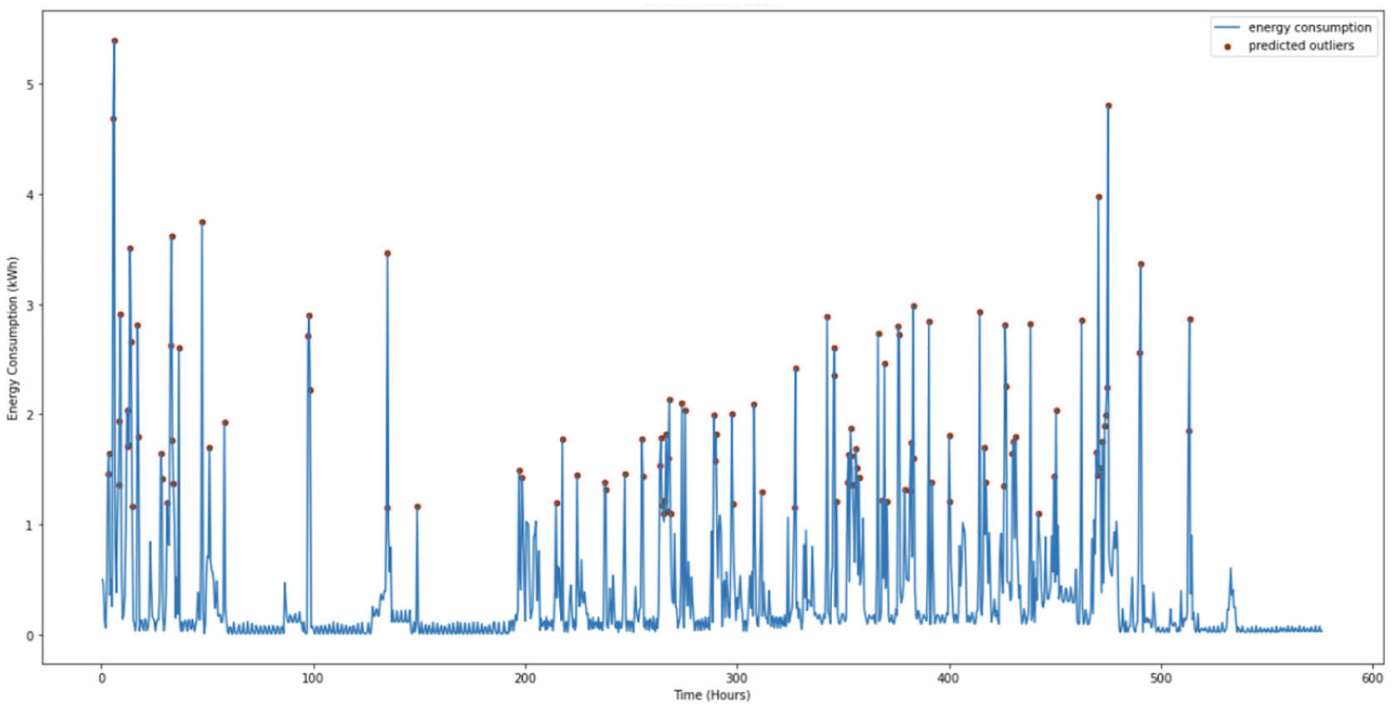

(a)

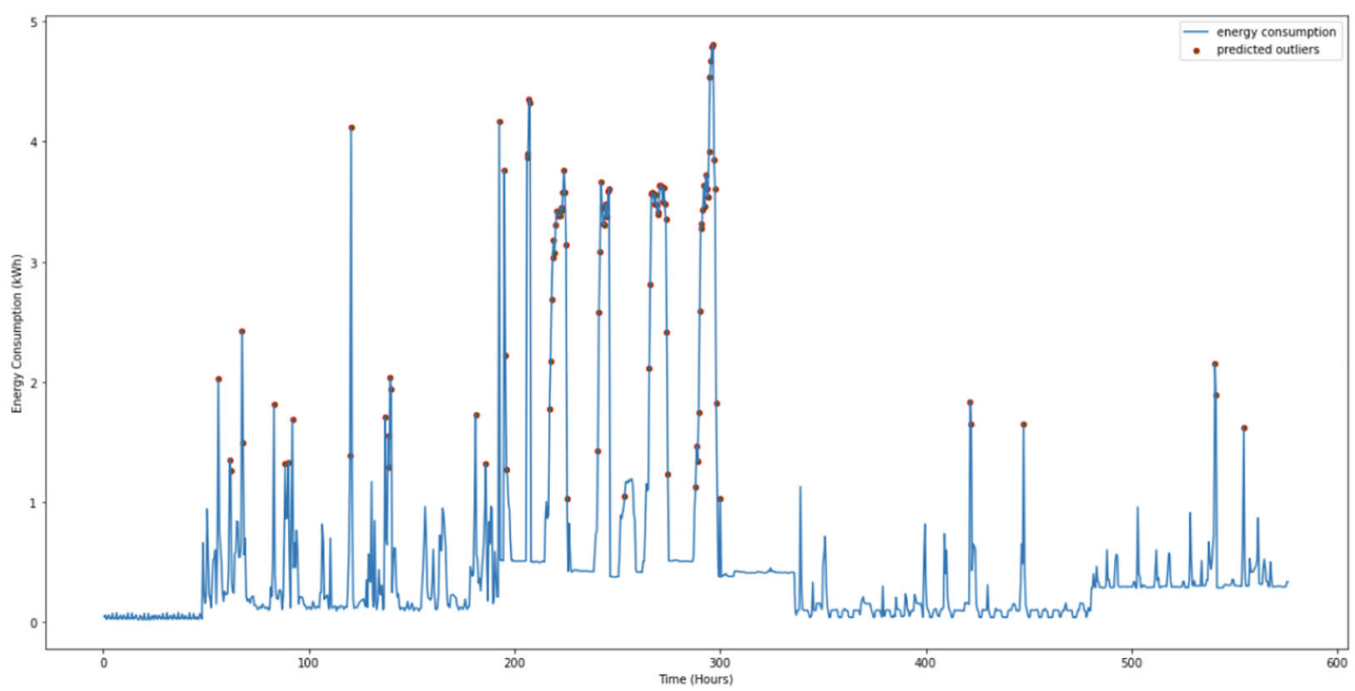

(b)

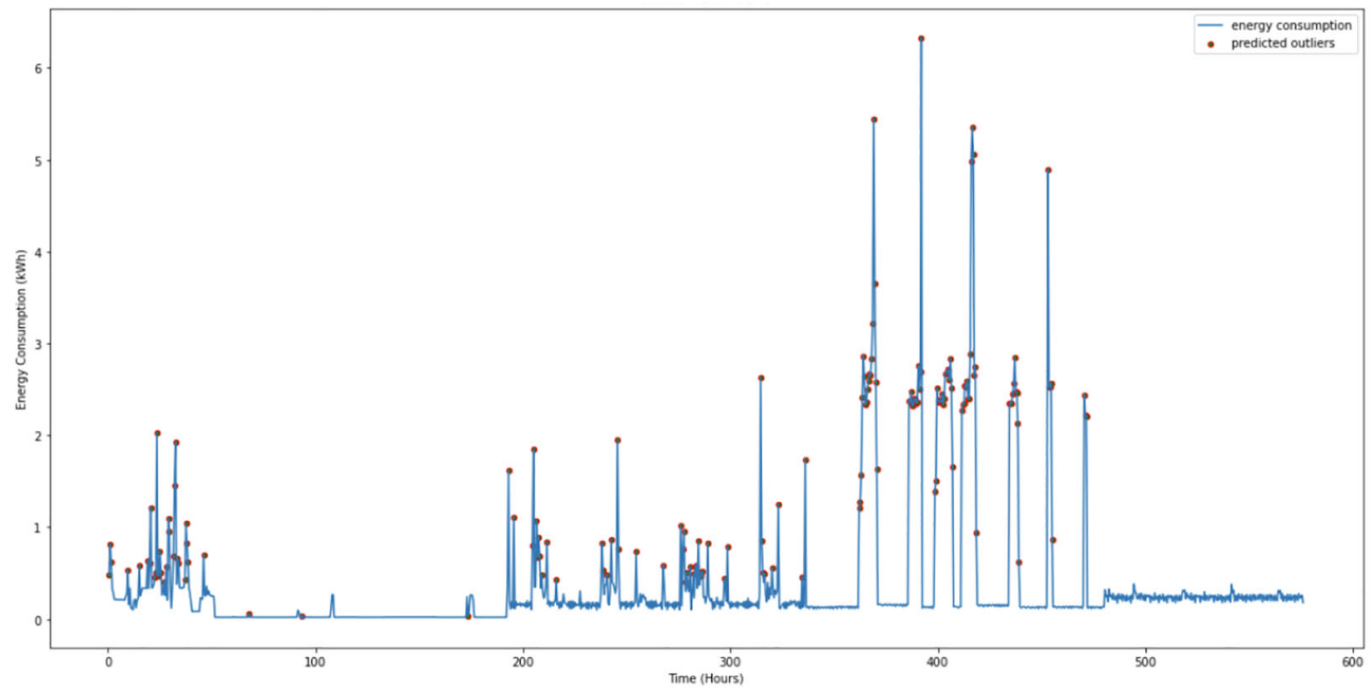

(c)

Figure 2. Energy curves of simulated energy consumption data and spikes labeled as abnormal: (a) smart meter $\boldsymbol{A}$ (b) smart meter $B$ (c) smart meter $C$. 
Table 1. Performance scores of anomalous detection model for different smart meters.

\begin{tabular}{|c|c|c|c|c|c|}
\hline & & \multicolumn{3}{|c|}{ Smart meter ID } & \multirow[b]{2}{*}{ Average } \\
\hline & & $\boldsymbol{A}$ & B & C & \\
\hline \multirow[t]{3}{*}{ Normal (when $P=0$ ) } & Precision & 1.00 & 0.98 & 0.96 & 0.98 \\
\hline & Recall & 0.97 & 1.00 & 1.00 & 0.99 \\
\hline & F1 score & 0.98 & 0.99 & 0.98 & 0.98 \\
\hline \multirow[t]{3}{*}{ Abnormal (when $P=1$ ) } & Precision & 0.85 & 1.00 & 1.00 & 0.95 \\
\hline & Recall & 1.00 & 0.92 & 0.79 & 0.90 \\
\hline & F1 score & 0.92 & 0.96 & 0.88 & 0.92 \\
\hline
\end{tabular}

Table 2. Balanced accuracy score of the anomalous detection model for different smart meters.

\begin{tabular}{|l|l|}
\hline Smart meter ID & Balanced accuracy score \\
\hline$A$ & 0.9827 \\
\hline$B$ & 0.9576 \\
\hline$C$ & 0.8947 \\
\hline
\end{tabular}

Table 1 summarizes the above scores for smart meter ID $A$, smart meter ID $B$ and smart meter ID $C$. The calculations were performed for two cases when the positive, $\mathrm{P}=0$ and when the positive, $\mathrm{P}=1$.

The time series dataset involved is known to have issues of imbalanced data, with a large portion of the data considered normal consumption. The performance of the proposed model is further evaluated by balanced accuracy, a well-known metric for imbalanced data. The balanced accuracy is given as:

$$
\text { balanced accuracy }=\frac{1}{2}\left(\frac{\mathrm{TP}}{\mathrm{P}}+\frac{\mathrm{TN}}{\mathrm{N}}\right)
$$

which is the average of true positive rate and true negative rate. Table 2 provides the calculated balanced accuracy score for 3 different smart meters.

\section{Discussion}

In the performed simulation, labeling based on Isolation Forest was performed to the given time series data to identify the anomalous instances. The red dots associated with the peaks of the energy profiles in Figure 2 refer to the identified anomalous points. It can be observed that the Isolation Forest approach was able to detect almost all the local maximum points, which coincided with the instances of peaks in the energy consumption profiles. This tallied with the requirement of anomalous energy detection, as the spikes are not merely tied to the magnitude in $\mathrm{kW}$, but mainly depend on the behavior of the profile in its neighborhood. Thereby, Isolation Forest is able to capture the peak regardless of the pattern exhibits in the dataset. Despite the fact that the peak's value is low in comparison to the rest of the overall dataset, it is still an atypical peak when compared to surrounding past measurements that are lower. Based on the simulation results, we can observe that Isolation Forest is a suitable strategy for auto labeling the smart meter readings. The approach is relatively direct to implement when compared to alternative approaches that define or predict the anomalies based on absolute magnitude analysis with mean and variance. ${ }^{15}$ Isolation Forest is especially valuable if the user's behavior changes with the seasons, such as when the energy readings are high during high production periods but low during low production periods. In summary, Isolation Forest is capable not only in detecting the point anomalies, but also in detecting the peak anomalies in contextual perspectives. However, if using Isolation Forest to classify anomalies requires processing an entire series of data in a specific window, this may not be feasible given the rapid growth of electricity consumption data. To that end, the Isolation Forest-labeled data is used to train a Naive Bayes model, which enables rapid classification of a single piece of data into normal or abnormal states without requiring previous points to be processed batch-wise as in Isolation Forest. For both classification results by Naïve Bayes model, the precision scores obtained for all smart meters were above 0.85 , the recall values were above 0.79 and the $\mathrm{F} 1$ scores were above 0.88 . The recall value of smart meter ID $C$ for $\mathrm{P}=1$ was 0.79 , which was the lowest score of all. This indicates that the model could only recognize $79 \%$ of the actual anomalous data, which could be related to the flattening trend of the energy consumption profiles, 
making it more difficult and challenging to identify spikes. In general, the performance of the anomalous detection models was satisfactory. The average F1 score was 0.98 and 0.92 , for normal and abnormal data, respectively. This indicates that the model has harmonic high performance for both precision and recall. The time series dataset involved is known to have the issues of imbalanced data, with a large portion of the data considered normal consumptions. The balanced accuracy score for all smart meters was above 0.8947 . Therefore, the proposed anomalous detection model not only detects the anomalous points successfully, but also classifies the normal points appropriately.

\section{Conclusions}

This paper proposed a two-stage anomalous detection model, combining the Isolation Forest and Gaussian Naïve Bayes. The performed simulations showed that a balanced accuracy score of at least 0.8947 was achieved. The Isolation Forest approach was used to label normal and abnormal data, and it successfully identified the abnormal spikes based on the behavior of the energy consumption pattern rather than just the magnitude of consumption. In detecting anomalies, the Gaussian Naïve Bayes gave a satisfactory performance in detecting the abnormal points as well as the normal points. The proposed approach combining Isolation Forest and Naive Bayes can be used to detect anomalies in a given time series of energy consumption. In comparison to using only the Isolation Forest for classification, this hybrid approach provides a straightforward method for labelling the data, which is particularly useful when the data set is large and arrives in batches. Classification does not have to be repeated using Isolation Forest and all available data; rather, once trained, the trained Naive Bayes model can classify effectively. However, the size of the current simulation was limited by the available dataset. A larger amount of data should be collected and applied to this model in the future. Furthermore, the optimum dataset size to achieve high accuracy also needs to be identified.

\section{Data availability}

Underlying data

Open Science Framework. Underlying data for 'Anomalous energy consumption detection using a Naïve Bayes approach', https://doi.org/10.17605/OSF.IO/CKTQS ${ }^{17}$

This project contains the following underlying data:

- Smart meter data.xlsx

\section{Software availability}

Archived source code at time of publication: https://doi.org/10.17605/OSF.IO/CKTQS ${ }^{17}$

This project contains the following parameter code:

- Model A GNB.ipynb

- Model B GNB.ipynb

- Model C GNB.ipynb

Data are available under the terms of the Creative Commons Attribution 4.0 International license (CC BY 4.0 Public domain dedication)

\section{References}

1. Horinov S, Horinova S: Energy management systems. GCSEEA Global Conference on Sustainable Environment, Energy and Agriculture (GCSEEA-2017). 2017. Publisher Full Text

2. Shapi MKM, Ramli NA, Awalin LI: Energy consumption prediction by using machine learning for smart building: Case study in Malaysia. Developments in the Built Environment. 2021; 5: 100037. Publisher Full Text

3. Ramli H, Ahmad SS, Abdullah A, et al.: Let's Get Smart. Energy Malaysia, Energy Commission Malaysia. 2019; 19: 8-15.
4. Himeur Y, Alsalemi A, Bensaali F, et al.: A novel approach for detecting anomalous energy consumption based on micro-moments and deep neural networks. Cogn. Comput. 2021; $1-23$.

5. Cui W, Wang $\mathrm{H}$ : Anomaly detection and visualization of school electricity consumption data. 2017 IEEE 2nd International Conference on Big Data Analysis (ICBDA). 2017; pp. 606-611. Publisher Full Text

6. Ma Z, Song J, Zhang J: A real-time detection method of abnormal building energy consumption data coupled POD-LSE and FCD. 
Procedia Eng. 2017; 205: 1657-1664.

Publisher Full Text

7. Mulongo J, Marcellin A, Theophilus A-S, et al:: Anomaly Detection in Power Generation Plants Using Machine Learning and Neural Networks. Appl. Artif. Intell. 2020; 34(1): 64-79.

Publisher Full Text

8. Manimaran A, Chandramohan D, Shrinivas S, et al.:

A comprehensive novel model for network speech anomaly detection system using deep learning approach. Int. J. Speech Technol. 2020; 23: 305-313.

Publisher Full Text

9. Aldweesh A, Derhab A, Emam AZ: Deep learning approaches for anomaly-based intrusion detection systems: A survey, taxonomy, and open issues. Knowl.-Based Syst. 2020; 189: 105124 Publisher Full Text

10. Chalapathy R, Chawla S: Deep learning for anomaly detection: A survey. arXiv:1901.03407. 2019.

11. Lin G, Claridge D: A temperature-based approach to detect abnormal building energy consumption. Energ. Buildings. 2015; 93: $110-118$. Publisher Full Text

12. Araya DB, Grolinger K, ElYamany HF, et al.: Collective contextual anomaly detection framework for smart buildings. 2016
International Joint Conference on Neural Networks (IJCNN). 2016; pp. 511-518.

Publisher Full Text

13. Liu X, Iftikhar N, Nielsen PS, et al.: Online anomaly energy consumption detection using lambda architecture. International Conference on Big Data Analytics and Knowledge Discovery. Porto: Springer; 2016; pp. 193-209.

14. Chen B, Sinn M, Ploennigs J, et al.: Statistical Anomaly Detection in Mean and Variation of Energy Consumption. $201422 n d$ International Conference on Pattern Recognition. 2014; pp. 3570-3575. Publisher Full Text

15. Himeur Y, Alsalemi A, Bensaali F, et al.: A Novel Approach for Detecting Anomalous Energy Consumption Based on MicroMoments and Deep Neural Networks. Cogn. Comput. 2020; 12 1381-1401.

Publisher Full Text

16. Liu FT, Ting KM, Zhou Z: Isolation Forest. 2008 Eighth IEEE International Conference on Data Mining. 2008; pp. 413-422. Publisher Full Text

17. Lim JY, Tan W-N, Tan Y-F: Anomalous energy consumption detection using a Naïve Bayes approach. Data and ipynb files. Open Science Framework. 2021.

Publisher Full Tex 


\section{Open Peer Review}

\section{Current Peer Review Status: ?}

\section{Version 1}

Reviewer Report 16 February 2022

https://doi.org/10.5256/f1000research.74260.r120393

(C) 2022 Tay K. This is an open access peer review report distributed under the terms of the Creative Commons Attribution License, which permits unrestricted use, distribution, and reproduction in any medium, provided the original work is properly cited.

\section{Kim Gaik Tay}

Faculty of Electrical and Electronic Engineering, Universiti Tun Hussein Onn Malaysia, Batu Pahat, Malaysia

This paper demonstrates the use of unsupervised machine learning techniques by Isolation Forest to label normal and anomalous points of energy consumption, as well as a Gaussian supervised method, namely Naïve Bayes, to classify the normal and anomalous points of energy consumption and predict the unseen anomalies from the smart meter reading. This work could be approved pending several polishes.

1. Methods section of Abstract. Please change "The proposed algorithm utilized supervised and unsupervised machine learning techniques, namely Isolation Forest and Gaussian Naïve Bayes" to "The proposed algorithm utilized unsupervised and supervised machine learning techniques, namely Isolation Forest and Gaussian Naïve Bayes." Because Isolation Forest is unsupervised while Naïve Bayes is supervised method.

2. In Results section of Abstract, "The data used were simulated data collected in less than a month with 30-minute reading intervals. The data were divided into testing and validation sets according to a ratio of 7:3." Is the data here training data? The training data was divided into $70 \%$ training and $30 \%$ testing?

3. The statement before Results section states that "The proposed anomalous detection model was verified using simulated smart meter readings with each reading for a smart meter collected in 30-minute intervals for a period of 576 hours. Firstly, the pattern of the electricity data of the Irish Social Science Data Archive (ISSDA) were investigated. Next, three sets of smart readings were generated based on different values of peak to-peak and valley-to-valley intervals. Then, the energy consumption values were manually created based on assumption of different users' behavior." Please clearly explain the training, testing and validation datasets and their ratio. Are training datasets from ISSDA and testing datasets generated manually? Are the $30-$ minutes intervals for a period of 576 hours testing data or training data? How is data generated based on peak to peak and valley-to-valley intervals? The abstract mentioned validation, but here no: please tally this section with the abstract. 
4. Explain in greater detail how Isolation Forest can label energy distribution as normal or anomalous? Also, how does one calculate $h(x)$ from $x$ ? What is $n, s(x, n)$ ? The equation to calculate anomaly score should labeled as (1).

5. Explain each term in Examples (1) and (2) such as $p(x=v \mid C k), p\left(C_{1} \mid x\right), p(x \mid C 1), p(C 1), p(x)$. How to calculate $v, p(x \mid C 1), p(C 1), p(x)$ ? Explain further what Bessel corrected variance is. What is the usage of Example (1) in classifying if a point is normal or abnormal?

6. Is Figure 2 training, validation or testing results? Where is normal point?

7. Since you define precision as TP/(TP+FP), and recall as TP/(TP+FN), then it is confusing for the reader that in Table 1, in the section for Normal (when $P=0$ ) that you use the labels Precision and Recall here as well. I would recommend writing Abnormal Precision as $=$ $\mathrm{TP} /(\mathrm{TP}+\mathrm{FP})$ and Normal Precision as TN/(TN + FN); Abnormal Recall as TP/(TP+FN ) and Normal Recall as TN/(TN + FP); and Abnormal F1 score $=2$. abnormal precision* abnormal recall/ (abnormal precision + abnormal recall) and normal F1 score $=2$. normal precision * normal recall/ (normal precision + normal recall).

8. What are $\mathrm{P}$ and $\mathrm{N}$ in balanced accuracy?

9. From Discussion part, line 7, "... it is still an "atypical" peak..." Any typo at "atypical"?

Is the work clearly and accurately presented and does it cite the current literature? Yes

Is the study design appropriate and is the work technically sound?

Yes

Are sufficient details of methods and analysis provided to allow replication by others? Partly

If applicable, is the statistical analysis and its interpretation appropriate? Yes

Are all the source data underlying the results available to ensure full reproducibility? Yes

Are the conclusions drawn adequately supported by the results? Yes

Competing Interests: No competing interests were disclosed.

Reviewer Expertise: Machine learning

I confirm that I have read this submission and believe that I have an appropriate level of expertise to confirm that it is of an acceptable scientific standard, however I have 
significant reservations, as outlined above.

The benefits of publishing with F1000Research:

- Your article is published within days, with no editorial bias

- You can publish traditional articles, null/negative results, case reports, data notes and more

- The peer review process is transparent and collaborative

- Your article is indexed in PubMed after passing peer review

- Dedicated customer support at every stage

For pre-submission enquiries, contact research@f1000.com 